\title{
Finite Time Step and Spatial Grid Effects in $\delta f$ Simulation of Warm Plasmas
}

\author{
Benjamin J. Sturdevant ${ }^{\mathrm{a}, \mathrm{b}, *}$, Scott E. Parker ${ }^{\mathrm{a}}$ \\ ${ }^{a}$ Department of Physics, University of Colorado at Boulder, Boulder, CO 80309, USA \\ ${ }^{b}$ Department of Applied Mathematics, University of Colorado at Boulder, Boulder, CO 80309, USA
}

\begin{abstract}
This paper introduces a technique for analyzing time integration methods used with the particle weight equations in $\delta f$ method particle-in-cell (PIC) schemes. The analysis applies to the simulation of warm, uniform, periodic or infinite plasmas in the linear regime and considers the collective behavior similar to the analysis performed by Langdon for full-f PIC schemes 1] 2. We perform both a time integration analysis and spatial grid analysis for a kinetic ion, adiabatic electron model of ion acoustic waves. An implicit time integration scheme is studied in detail for $\delta f$ simulations using our weight equation analysis and for full- $f$ simulations using the method of Langdon. It is found that the $\delta f$ method exhibits a CFL-like stability condition for low temperature ions, which is independent of the parameter characterizing the implicitness of the scheme. The accuracy of the real frequency and damping rate due to the discrete time and spatial schemes is also derived using a perturbative method. The theoretical analysis of numerical error presented here may be useful for the verification of simulations and for providing intuition for the design of new implicit time integration schemes for the $\delta f$ method, as well as understanding differences between $\delta f$ and full- $f$ approaches to plasma simulation.
\end{abstract}

Keywords: $\delta f$ Method, Plasma Simulation, Particle-in-Cell, Numerical Dispersion, Numerical Stability, Time Integration, Implicit Methods

\section{Introduction}

The $\delta f$ method for particle simulation was developed in the nineties as a way to reduce the noise from particle-in-cell (PIC) methods introduced by using discrete particles to represent continuous distribution functions over phase space [3] [4] 5] [6] [7]. In this algorithm, the full particle distribution function $f$ is separated into a "background" equilibrium part $f_{0}$, which is known analytically, and a perturbed part $\delta f$ which is solved numerically by evolving the equations of motion along with a particle "weight" equation for a finite number of computational particles. Previous studies regarding the numerical properties of the $\delta f$ method have focused on its sampling noise properties [6] [5, but to the authors' knowledge, comprehensive methods for evaluating the effects introduced by the discretization schemes used with the $\delta f$ method have not previously been developed.

In this paper, we introduce a technique, motivated by the work of Langdon [1], to analyze the time integration scheme used in the $\delta f$ method for a uniform, warm, periodic or infinite plasma in the linear regime. In the analysis, we consider the evolution of the particle weights at discrete time increments along the unperturbed particle trajectories. An end point condition on the particle motion establishes a connection between the value of $\delta f$ along the characteristic followed by a Lagrangian particle and the value of $\delta f$ at a fixed Eulerian point in phase space. The related Eulerian equation for $\delta f$ is valid when the scheme has

\footnotetext{
${ }^{*}$ Corresponding author

Email address: benjamin.j.sturdevant@gmail.com (Benjamin J. Sturdevant)
} 
converged in the number of computational particles, and it includes the effects of the finite time step. An explicit expression for $\delta f$ is obtained from the Eulerian equation by assuming a von Neumann-like ansatz for all spatial and time varying quantities.

To illustrate the use of this technique, we perform a complete analysis of an implicit integration scheme applied to a kinetic ion, adiabatic electron plasma model which allows the propagation of ion acoustic waves. The choice to study this particular model is motivated by its relation to the hybrid Lorentz force ion and fluid electron model developed in 8]. The ion acoustic wave model presented here is a good test bed for further development of the Lorentz ion hybrid model [9] [10] [11. In fact, the model presented here can be trivially extended to model the ion-temperature gradient instability [12. Here, we simply lay out the method for numerical analysis of the $\delta f$ method using the test problem important in our particular application area. This analysis can be generalized and applied to other physical models, for example, to analyze the numerical error associated with $\delta f$ drift-kinetic electrons in gyrokinetic turbulence simulations. It is shown that the numerical properties of the $\delta f$ method applied to the test problem are independent of a parameter characterizing the implicitness of the integration scheme. The time integration analysis is then combined with the spatial grid analysis of Langdon 2. A modified dispersion relation including effects of both $\Delta t$ and $\Delta x$ is obtained from which the lowest order corrections to the real frequency and damping rate are found. Numerical solutions of the modified dispersion relation are also used to study the stability of the simulation model, and a CFL-like stability condition is found for the $\delta f$ method when the ion temperature is low. To validate our results, simulations are performed and compared to the theory.

In analyzing the numerical properties of the $\delta f$ method, one of our goals is to assess differences from the conventional full- $f$ method. To address this, we compare the $\delta f$ model with a full- $f$ formulation for the same model problem, including the same implicit integration scheme. Following the analysis of Langdon, we obtain the modified dispersion relation for the full- $f$ method. Some comments should be made regarding the linear analysis used to make comparisons of the two methods. In the full- $f$ method, the linear correction to the number density due to a small electric field is introduced through the perturbed particle orbits. In the $\delta f$ method, however, the linear correction is introduced through the particle weights, and the perturbed particle orbits account for a correction at a higher order. Hence, the time integration scheme used for the particle weight equations determine the finite time step effects on the linear dispersion relation for the $\delta f$ method, whereas the time integration scheme used for the equations of motion determine the finite time step effects on the linear dispersion relation for the full- $f$ method.

The paper is organized as follows. In Section 2, we present the $\delta f$ and full- $f$ methods for Particle-in-Cell (PIC) simulations of a kinetic ion species and discuss the linearization of both methods. In Section 3, a field equation is introduced to close the simulation model, assuming adiabatic electrons and quasi-neutrality. The closed system allows for ion Landau damping of ion acoustic waves. In Section 4, our time integration analysis for the $\delta f$ method is presented and applied to the implicit scheme used to advance the ions. The time integration analysis of Langdon is applied to the same implicit scheme used with the particle equations of motion in the full- $f$ method in order to make comparisons. In Section 5, the spatial grid analysis of Langdon is applied to both the $\delta f$ and full- $f$ methods to derive modified dispersion relations including the combined effects of $\Delta t$ and $\Delta x$. In Section 6 , the modified dispersion relations are studied in detail, and results regarding the accuracy and stability of the numerical methods are obtained. Simulations are performed and used to validate the numerical analysis.

\section{Full- $f$ and $\delta f$ Particle-in-Cell Methods for Vlasov Ions}

We consider the Vlasov equation to describe the evolution of the distribution function $f$ for a uniform, unmagnetized ion species with charge $e$

$$
\frac{\partial f}{\partial t}+\mathbf{v} \cdot \nabla f+\frac{e}{m_{i}} \mathbf{E} \cdot \nabla_{\mathbf{v}} f=0
$$

In this section, we present the equations used for both the full- $f$ and $\delta f$ methods for PIC simulation of Eq.(1). These methods are both based on evolving a system of $N_{p}$ computational particles through phase 
space along the characteristics of Eq.(1). We consider simulations in which the objective of the PIC model is to obtain a number density which is coupled to a field equation for E. A specific example of such a field equation is given in Section 3 and studied in detail for an implicit time integration scheme applied to the PIC model. The linearization of both PIC methods will be discussed at the end of this section.

\subsection{Full-f Method}

We first present the familiar full- $f$ method for solving Eq. 11. This method is discussed in a number of references including 13 and $[14$. The computational particles (ions) are taken to follow the Newton-Lorentz equations of motion

$$
\begin{aligned}
\frac{d \mathbf{x}_{p}(t)}{d t} & =\mathbf{v}_{p}(t) \\
\frac{d \mathbf{v}_{p}(t)}{d t} & =\frac{e}{m_{i}} \mathbf{E}\left(\mathbf{x}_{p}(t), t\right)
\end{aligned}
$$

for $p=1, \ldots, N_{p}$, and the corresponding distribution function is given by a Klimontovich representation

$$
f(\mathbf{x}, \mathbf{v}, t)=\sum_{p=1}^{N_{p}} \delta_{X}\left(\mathbf{x}-\mathbf{x}_{p}(t)\right) \delta_{V}\left(\mathbf{v}-\mathbf{v}_{p}(t)\right) .
$$

Here $\delta_{X}$ and $\delta_{V}$ are scaled Dirac delta functions with units of length ${ }^{-d_{x}}$ and velocity ${ }^{-d_{v}}$ respectively, where $d_{x}$ and $d_{v}$ are the spatial and velocity dimensions for the simulation. A normalization factor of $N_{s} / N_{p}$ is also implicit in the Klimontovich representation, where $N_{s}$ is the number of physical particles to be modeled. Hence, integrating $f$ over all phase space will yield $N_{s}$. It can be verified that Eq.(3) satisfies Eq. (1) when the computational particles evolve according to Eq.(2) and derivatives are taken in a distributional sense. By integrating Eq. (3) over velocity space, we have the following expression for the number density

$$
n(\mathbf{x}, t)=\sum_{p=1}^{N_{p}} \delta_{X}\left(\mathbf{x}-\mathbf{x}_{p}(t)\right) .
$$

In PIC methods, $\delta_{X}$ is approximated by a function supported on a set of finite measure, which is used to obtain the grid number density from the computational particles. The approximation of $\delta_{X}$ is called the shape function and is denoted by $S$. The PIC approximation of Eq. (4) at grid point $\mathbf{X}_{j}$ is then

$$
n_{j}(t) \approx \sum_{p=1}^{N_{p}} S\left(\mathbf{X}_{j}-\mathbf{x}_{p}(t)\right)
$$

Typically, the same function $S$ is used in Eq. 22 to interpolate the electric field values on grid points to a particle's location as

$$
\mathbf{E}\left(\mathbf{x}_{p}(t), t\right) \approx|\Delta \mathbf{x}| \sum_{j} \mathbf{E}_{j}(t) S\left(\mathbf{X}_{j}-\mathbf{x}_{p}(t)\right)
$$

\section{2. $\delta f$ Method}

The $\delta f$ method starts with the assumption that $f$ can be separated into a known time independent equilibrium part and an unknown perturbed part as $f=f_{0}+\delta f$. Particle weights are defined for each computational particle as

$$
w_{p}(t) \equiv \frac{\delta f\left(\mathbf{x}_{p}(t), \mathbf{v}_{p}(t), t\right)}{f\left(\mathbf{x}_{p}(t), \mathbf{v}_{p}(t), t\right)} .
$$


The positions and velocities of the computational particles are taken to follow Eq. (2), as in the full- $f$ method, and the weights are evolved according to

$$
\frac{d w_{p}(t)}{d t}=-\left.\left(1-w_{p}(t)\right) \frac{e}{m_{i}} \mathbf{E}\left(\mathbf{x}_{p}(t), t\right) \cdot \nabla_{\mathbf{v}} \ln f_{0}(\mathbf{v})\right|_{\mathbf{v}_{p}(t)},
$$

where the approximation Eq. (6) is used in the PIC model. The distribution function can then be represented as

$$
f(\mathbf{x}, \mathbf{v}, t)=f_{0}(\mathbf{v})+\sum_{p=1}^{N_{p}} w_{p}(t) \delta_{X}\left(\mathbf{x}-\mathbf{x}_{p}(t)\right) \delta_{V}\left(\mathbf{v}-\mathbf{v}_{p}(t)\right),
$$

which can be shown to satisfy Eq.(1) when the computational particles evolve according to Eq. 22 and the weights according to Eq. (8). An integration of Eq. (9) over velocity space yields for the number density

$$
n(\mathbf{x}, t)=n_{0}+\sum_{p=1}^{N_{p}} w_{p}(t) \delta_{X}\left(\mathbf{x}-\mathbf{x}_{p}(t)\right),
$$

which for the PIC method, is approximated at a grid point by

$$
n_{j}(t) \approx n_{0}+\sum_{p=1}^{N_{p}} w_{p}(t) S\left(\mathbf{X}_{j}-\mathbf{x}_{p}(t)\right)
$$

\subsection{Linearization of the PIC models}

The time stepping analysis for both the full- $f$ and $\delta f$ methods requires a linearization of the PIC model equations with respect to a small perturbation from equilibrium. This is accomplished for the full- $f$ method by splitting $\mathbf{x}_{p}$ and $\mathbf{v}_{p}$ into unperturbed and perturbed parts as

$$
\begin{aligned}
& \mathbf{x}_{p}=\mathbf{x}_{p}^{(0)}+\mathbf{x}_{p}^{(1)} \\
& \mathbf{v}_{p}=\mathbf{v}_{p}^{(0)}+\mathbf{v}_{p}^{(1)}
\end{aligned}
$$

where quantities with a superscript ${ }^{(0)}$ are independent of the electric field. The unperturbed particle orbit is then

$$
\mathbf{x}_{p}^{(0)}(t)=\mathbf{x}_{p}^{(0)}(t=0)+t \mathbf{v}_{p}^{(0)}
$$

with $\mathbf{v}_{p}^{(0)}$ constant in time. The linear correction to the particle orbit is given by

$$
\begin{aligned}
& \frac{d \mathbf{x}_{p}^{(1)}(t)}{d t}=\mathbf{v}_{p}^{(1)}(t) \\
& \frac{d \mathbf{v}_{p}^{(1)}(t)}{d t}=\frac{e}{m_{i}} \mathbf{E}^{(1)}\left(\mathbf{x}_{p}^{(0)}(t), t\right)
\end{aligned}
$$

where $\mathbf{E}^{(1)}$ is self consistent with the linear approximation to the number density $n_{j}(t) \approx n_{0}+\delta n_{j}^{(1)}(t)$, where $\delta n_{j}^{(1)}(t)$ is given by

$$
\delta n_{j}^{(1)}(t)=-\nabla \cdot \sum_{p=1}^{N_{p}} \mathbf{x}_{p}^{(1)}(t) S\left(\mathbf{X}_{j}-\mathbf{x}_{p}^{(0)}(t)\right) .
$$

\section{This is the linearized trajectory method as described in 15 .}

For the $\delta f$ method, the linear correction comes into the number density through the particle weights rather than through the perturbed particle orbits. Details on the linearization of the $\delta f$ PIC model can 
be found in Appendix A. Assuming $\mathbf{x}_{p}^{(0)}$ satisfies Eq. 13 with $\mathbf{v}_{p}^{(0)}$ constant, the linear particle weight $w_{p}^{(1)}$ evolves as

$$
\frac{d w_{p}^{(1)}(t)}{d t}=-\left.\frac{e}{m_{i}} \mathbf{E}^{(1)}\left(\mathbf{x}_{p}^{(0)}(t), t\right) \cdot \nabla_{\mathbf{v}} \ln f_{0}(\mathbf{v})\right|_{\mathbf{v}_{p}^{(0)}} .
$$

Here $\mathbf{E}^{(1)}$ is the electric field that is self consistent with the linear approximation to the number density $n_{j}(t) \approx n_{0}+\delta n_{j}^{(1)}(t)$, where $\delta n_{j}^{(1)}(t)$ is given by

$$
\delta n_{j}^{(1)}(t)=\sum_{p=1}^{N_{p}} w_{p}^{(1)}(t) S\left(\mathbf{X}_{j}-\mathbf{x}_{p}^{(0)}(t)\right)
$$

and the following definition for $w_{p}^{(1)}$ applies as a result of linearization:

$$
w_{p}^{(1)}(t)=\frac{\delta f\left(\mathbf{x}_{p}^{(0)}(t), \mathbf{v}_{p}^{(0)}, t\right)}{f_{0}\left(\mathbf{v}_{p}^{(0)}\right)} .
$$

When considering the discrete time PIC method, it should be kept in mind that modifications to the linear dispersion relation in the full- $f$ method due to finite $\Delta t$ result from the integration scheme used for the particle equations of motion, whereas in the $\delta f$ method, modifications result from the integration scheme used for the particle weight equation. The corrections due to the perturbed particle orbits come in at a higher order in the $\delta f$ method, and therefore any consistent integration scheme applied to the particle equations of motion should produce similar results at least for simulations running in the linear regime. Both PIC methods when linearized, provide approximations to the linearized Vlasov equation. The linearized Vlasov equation is simply given by

$$
\frac{\partial \delta f}{\partial t}+\mathbf{v} \cdot \nabla \delta f+\frac{e}{m_{i}} \mathbf{E}^{(1)} \cdot \nabla_{\mathbf{v}} f_{0}=0 .
$$

\section{Model for Electrons and Electric Field}

For our analysis, we consider an adiabatic electron model, which when combined with a quasi-neutrality assumption, will provide an equation coupling the electrostatic $\mathbf{E}$ field with $\delta n \equiv n-n_{0}$, to close our simulation model. Linearization of a Boltzmann distribution yields for the perturbed electron number density

$$
\delta n_{e}(\mathbf{x}, t)=n_{0} \frac{e \phi(\mathbf{x}, t)}{T_{e}}
$$

where $-e$ is the electron charge and $T_{e}$ the electron temperature taken to be constant. Specifying $\mathbf{E}=-\nabla \phi$ and assuming quasi-neutrality $\delta n_{e}=\delta n_{i} \equiv \delta n$, we have

$$
\mathbf{E}(\mathbf{x}, t)=-\frac{T_{e}}{e} \frac{\nabla \delta n(\mathbf{x}, t)}{n_{0}} .
$$

Our model is then closed from $\delta n$ obtained directly from the ions, which for the linear PIC models are the second terms on the right hand sides of Eq.(15) and Eq.(17). We chose this field model to study because it is the electrostatic limit of the generalized Ohm's law derived in 8 for the kinetic ion, fluid electron model. This reduced model allows for the propagation and ion Landau damping of ion acoustic waves. A dispersion relation from Vlasov theory can be derived using Eq.(19) and Eq.21], which is used to compare the dispersion of our PIC models. For an arbitrary equilibrium distribution $f_{0}$, the dispersion relation is

$$
\epsilon_{0}(\omega, \mathbf{k}) \equiv 1-\frac{T_{e}}{m_{i} n_{0}} \mathbf{k} \cdot \int \frac{\nabla_{\mathbf{v}} f_{0}(\mathbf{v})}{\mathbf{k} \cdot \mathbf{v}-\omega} d \mathbf{v}=0 .
$$


in Eq. (26), which gives for the unperturbed particle orbit

$$
\mathbf{x}_{\nu}^{(0)}=\mathbf{x}+(\nu-N) \Delta t \mathbf{v} .
$$

$$
\epsilon_{0}(\zeta)=1-\frac{1}{2 T} Z^{\prime}(\zeta / \sqrt{2})=0
$$
Fried and Conte [16, defined by the complex integral

$$
Z(\theta)=\frac{1}{\sqrt{\pi}} \int_{-\infty}^{\infty} \frac{e^{-s^{2}}}{(s-\theta)} d s, \quad \operatorname{Im} \theta>0
$$

and its analytic continuation for $\operatorname{Im} \theta \leq 0$.

\section{Analysis of an Implicit Time Integration Scheme} Picard iteration scheme or with a Jacobian-free Newton-Krylov solver [18 [19.

$$
\mathbf{x}_{\nu}^{(0)}=\mathbf{x}_{\nu=0}^{(0)}+\nu \Delta t \mathbf{v}^{(0)}
$$
that the time discretized unperturbed orbits can be solved analytically.

$$
\begin{aligned}
& \mathbf{x}_{\nu=N}^{(0)}=\mathbf{x} \\
& \mathbf{v}_{\nu=N}^{(0)}=\mathbf{v}
\end{aligned}
$$

where we define $v_{t h} \equiv \sqrt{T_{i} / m_{i}}$ with $T_{i}$ being the ion temperature. In this case, we have

defining the parameters $T$ to be the ratio of ion and electron temperatures, $T=T_{i} / T_{e}$, and $\zeta$ to be the phase velocity normalized by the ion thermal velocity, $\zeta=\omega / k v_{t h}$. Here $Z(\theta)$ is the plasma dispersion function of

In analyzing the finite time step and spatial grid effects, discrete particle effects are ignored, assuming convergence in the number of computational particles. For the remainder of the paper, we drop the subscript $p$ from particle quantities, since there isn't a need to distinguish between individual particles in the analysis. A subscript $\nu$ is now used instead to index a quantity evaluated at time step $\nu \Delta t$. We consider the implicit ODE time integration scheme used in $[8$ with a time centering parameter $\alpha$. The scheme is convergent for $0 \leq \alpha \leq 1$ and yields the familiar methods of forward Euler, trapezoidal, and backward Euler for the values of $\alpha=0, \alpha=1 / 2$, and $\alpha=1$ respectively. It is a first order accurate ODE integration method for all values of $\alpha$ except for the special case of $\alpha=1 / 2$, which is second order accurate. See for example, Chapter 6 of [17. It is implicit for all $\alpha$ except for $\alpha=0$. The implicit equations can be solved, for example, through a

The time integration analysis can be regarded as either an exact analysis of the scheme applied to the linearized PIC models described in Section 2.3 or as an approximate analysis for the nonlinear PIC methods when initialized by a small perturbation and run for a short time. The unperturbed particle orbits in our model will be reproduced exactly due to the consistency of the integration scheme, yielding

where $\mathbf{v}^{(0)}$ is constant in time. The unperturbed velocity being constant in time allows for a simple analysis. The analysis can be extended to more complex models, for example a magnetized plasma model, provided

Since we are interested in the evolution of the distribution function rather than the individual particle trajectories, we will make the connection between a Lagrangian particle's phase space location at time step $\nu$ and the Eulerian phase space coordinates. Suppose we wish to know the distribution function at the discrete time step $\nu=N$. Taking a fixed, arbitrary point $(\mathbf{x}, \mathbf{v})$, we consider a particle with an unperturbed orbit which passes through this point and enforce that it does so at $\nu=N$. In particular, we require that 
This can be thought of as choosing the particles with initial conditions such that they will contribute to the density in the infinitesimal region around $(\mathbf{x}, \mathbf{v})$ at time step $\nu=N$. It assumes convergence in the number of computational particles, so we can assume a continuous distribution function. Since the point (x, v) and time $\nu=N$ were chosen arbitrarily, knowing the distribution function for these arguments will give the distribution function over all of phase space and at all discrete time values.

\subsection{Time Integration Analysis for the $\delta f$ Method}

Applying the time integration scheme from [8] to the $\delta f$ method yields the following discrete equation for the linear particle weights

$$
\frac{w_{\nu}^{(1)}-w_{\nu-1}^{(1)}}{\Delta t}=-(1-\alpha) G_{\nu-1}^{(1)}\left(\mathbf{x}_{\nu-1}^{(0)}, \mathbf{v}^{(0)}\right)-\alpha G_{\nu}^{(1)}\left(\mathbf{x}_{\nu}^{(0)}, \mathbf{v}^{(0)}\right)
$$

where we have defined

$$
G_{\nu}^{(1)}\left(\mathbf{x}_{\nu}^{(0)}, \mathbf{v}^{(0)}\right) \equiv \frac{e}{m_{i}} \mathbf{E}_{\nu}^{(1)}\left(\mathbf{x}_{\nu}^{(0)}\right) \cdot \nabla_{\mathbf{v}^{(0)}} \ln f_{0}\left(\mathbf{v}^{(0)}\right)
$$

Noting the definition for $w^{(1)}$ in Eq. 18 and using the Eulerian referenced particle expression in Eq. 28, we obtain an Eulerian equation relating $\delta f$ at the discrete time steps $\nu=N$ and $\nu=N-1$

$$
\begin{aligned}
& \delta f_{N}(\mathbf{x}, \mathbf{v})=\delta f_{N-1}(\mathbf{x}-\Delta t \mathbf{v}, \mathbf{v})- \\
& \Delta t\left[(1-\alpha) G_{N-1}^{(1)}(\mathbf{x}-\Delta t \mathbf{v}, \mathbf{v})+\alpha G_{N}^{(1)}(\mathbf{x}, \mathbf{v})\right] f_{0}(\mathbf{v})
\end{aligned}
$$

Next, to obtain an explicit expression for $\delta f$, we take a von-Neumann-like ansatz, assuming that $\delta f_{N}$ and $G_{N}^{(1)}$ have time and spatial dependence as

$$
\delta f_{N}(\mathbf{x}, \mathbf{v})=\delta f(\mathbf{k}, \mathbf{v}, z) z^{N} e^{i \mathbf{k} \cdot \mathbf{x}} \quad G_{N}^{(1)}(\mathbf{x}, \mathbf{v})=G^{(1)}(\mathbf{k}, \mathbf{v}, z) z^{N} e^{i \mathbf{k} \cdot \mathbf{x}} .
$$

Here $z$ is the amplification factor and can be written in the more familiar form $z=e^{-i \omega \Delta t}$ for a complex frequency $\omega$. Putting the ansatz into Eq. 31) and using the definition of $G^{(1)}$, we obtain

$$
\delta f(\mathbf{k}, \mathbf{v}, z)=\frac{e}{m_{i}}\left[\frac{\Delta t}{1-z e^{i \mathbf{k} \cdot \mathbf{v} \Delta t}}-\alpha \Delta t\right] \mathbf{E}^{(1)}(\mathbf{k}, z) \cdot \nabla_{\mathbf{v}} f_{0}(\mathbf{v}) .
$$

Finally, an integration over velocity provides an expression for the linear perturbed number density

$$
\delta n^{(1)}(\mathbf{k}, z)=\frac{e}{m_{i}} \mathbf{E}^{(1)}(\mathbf{k}, z) \cdot \int\left[\frac{\Delta t}{1-z e^{i \mathbf{k} \cdot \mathbf{v} \Delta t}}\right] \nabla_{\mathbf{v}} f_{0}(\mathbf{v}) d \mathbf{v},
$$

which will be used in the spatial grid analysis and coupled to our field model in the following section.

It is interesting to note that for sufficiently well behaved $f_{0}$, the $\alpha \Delta t$ term in Eq. (33) does not contribute to the integral. The perturbed number density, Eq.(34), and therefore the modified dispersion relation will be independent of the time centering parameter $\alpha$. In this paper, the model is electrostatic, therefore only $\delta n$ is needed in the field equation. If higher velocity moments are needed, for example in an electromagnetic model, $\alpha$ is expected to have important effects on the numerical properties of the PIC model. Multiplying Eq. (33) by $\mathbf{v}$ and integrating over velocity yields for the perturbed flux density

$$
\delta n \mathbf{u}^{(1)}(\mathbf{k}, z)=\frac{e}{m_{i}} \int \mathbf{v}\left[\frac{\Delta t}{1-z e^{i \mathbf{k} \cdot \mathbf{v} \Delta t}}\right] \mathbf{E}^{(1)}(\mathbf{k}, z) \cdot \nabla_{\mathbf{v}} f_{0}(\mathbf{v}) d \mathbf{v}+\alpha \Delta t \frac{e}{m_{i}} n_{0} \mathbf{E}^{(1)}(\mathbf{k}, z),
$$

showing that the $\alpha$ dependence does not necessarily vanish for velocity moments other than $\delta n$.

Continuing with our electrostatic model, for $f_{0}=f_{M}$ in one dimension we have

$$
\delta n^{(1)}(k, \omega)=\frac{i e n_{0}}{2 k T_{i}} E^{(1)}(k, \omega) X_{\delta f}\left(\frac{\omega}{\sqrt{2} k v_{t h}} ; \sqrt{2} k v_{t h} \Delta t, 0\right)
$$


where $X_{\delta f}$ is defined by the complex integral

$$
X_{\delta f}(\theta ; \eta, \mu)=\frac{1}{\sqrt{\pi}} \int_{-\infty}^{\infty}\left[\frac{i \eta}{e^{i \eta[s(1-\mu)-\theta]}-1}\right] \frac{d}{d s} e^{-s^{2}} d s, \quad \operatorname{Im} \theta>0
$$

and its analytic continuation for $\operatorname{Im} \theta \leq 0$. It is assumed that the arguments $\eta$ and $\mu$ are real. Note that $X_{\delta f}(\theta ; \eta, 0) \rightarrow Z^{\prime}(\theta)$ as $\eta \rightarrow 0$, giving the result obtained from Vlasov theory Eq. (24) as $\Delta t \rightarrow 0$.

\subsection{Time Integration Analysis for the Full-f Method}

Next, we will perform Langdon's time integration analysis for the implicit scheme applied to the full- $f$ method. We will use this for making comparisons with the $\delta f$ method and refer the reader to [1] for details of how the analysis is performed. The time integration scheme applied to the full- $f$ method yields for the linear perturbed particle orbits

$$
\begin{aligned}
& \frac{\mathbf{x}_{\nu}^{(1)}-\mathbf{x}_{\nu-1}^{(1)}}{\Delta t}=(1-\alpha) \mathbf{v}_{\nu-1}^{(1)}+\alpha \mathbf{v}_{\nu}^{(1)} \\
& \frac{\mathbf{v}_{\nu}^{(1)}-\mathbf{v}_{\nu-1}^{(1)}}{\Delta t}=(1-\alpha) \frac{e}{m_{i}} \mathbf{E}_{\nu-1}^{(1)}\left(\mathbf{x}_{\nu-1}^{(0)}\right)+\alpha \frac{e}{m_{i}} \mathbf{E}_{\nu}^{(1)}\left(\mathbf{x}_{\nu}^{(0)}\right)
\end{aligned}
$$

The linear perturbed number density that results is

$$
\delta n^{(1)}(\mathbf{k}, z)=\frac{-i e}{m_{i}} \mathbf{E}^{(1)}(\mathbf{k}, z) \cdot \mathbf{k} \int\left[\frac{\Delta t}{1-z e^{i \mathbf{k} \cdot \mathbf{v} \Delta t}}-\alpha \Delta t\right]^{2} f_{0}(\mathbf{v}) d \mathbf{v} .
$$

For the one dimensional Maxwellian, this gives

$$
\delta n^{(1)}(k, \omega)=\frac{i e n_{0}}{2 k T_{i}} E^{(1)}(k, \omega) X_{f}\left(\frac{\omega}{\sqrt{2} k v_{t h}} ; \sqrt{2} k v_{t h} \Delta t, 0, \alpha\right)
$$

where $X_{f}$ is defined by the complex integral

$$
X_{f}(\theta ; \eta, \mu, \alpha) \equiv \frac{1}{\sqrt{\pi}}(1-\mu) \int_{-\infty}^{\infty}\left[\frac{i \eta}{e^{i \eta[s(1-\mu)-\theta]}-1}+i \alpha \eta\right]^{2} e^{-s^{2}} d s, \quad \operatorname{Im} \theta>0
$$

and its analytic continuation for $\operatorname{Im} \theta \leq 0$. It is assumed that the arguments $\eta, \mu$ and $\alpha$ are real. Again, we have that $X_{f}(\theta ; \eta, 0, \alpha) \rightarrow Z^{\prime}(\theta)$ as $\eta \rightarrow 0$, yielding the result obtained from Vlasov theory Eq.24.

\section{Finite Time Step and Spatial Grid Dispersion Relations}

In this section, we combine the time integration analyses for the $\delta f$ and full- $f$ methods with the spatial grid analysis in 2 to obtain modified dispersion relations for our model problem which include the effects of both $\Delta t$ and $\Delta x$ for a one dimensional periodic spatial grid. We begin by summarizing the main ideas of the spatial grid analysis and discuss the appropriate transforms for simulations on a grid with period $L_{x}$.

\subsection{Transforms for a Periodic Grid}

For the analysis, we must consider both continuum and discrete quantities over the spatial domain and their Fourier representations. The Fourier representation of a continuum quantity $P(x)$ is to be interpreted as a Fourier series (FS) coefficient. The transform pair is given by

$$
\begin{aligned}
& P(x)=\frac{1}{L_{x}} \sum_{k} P(k) e^{i k x} \\
& P(k)=\int_{0}^{L_{x}} P(x) e^{-i k x} d x,
\end{aligned}
$$


where the sum in Eq. 43 is over an infinite number of allowed values of $k$. The allowed values for a FS are $k=2 \pi m / L_{x}$ for each $m \in \mathbb{Z}$. In our model, the continuum quantities include the linear perturbed number density of point particles $\delta n^{(1)}(x)$, the electric field interpolated to the particle locations $E^{(1)}(x)$, and the shape function $S(x)$.

A discrete quantity $G_{j}$, which is defined on the grid points, has a discrete Fourier transform (DFT). The transform pair is given by

$$
\begin{aligned}
G_{j} & =\frac{1}{L_{x}} \sum_{k} G(k) e^{i k X_{j}} \\
G(k) & =\sum_{j=0}^{N_{x}-1} G_{j} e^{-i k X_{j}} \Delta x,
\end{aligned}
$$

where $N_{x}$ is the number of grid points and the sum in Eq.45 is over a finite number of allowed values of $k$. The allowed values of $k$ in a DFT are $k=2 \pi m / L_{x}$ for each $m \in\left\{-N_{x} / 2+1,-N_{x} / 2+2, \ldots, 0,1, \ldots N_{x} / 2\right\}$. The discrete quantities in our model include the perturbed number density of finite sized particles sampled on grid point, denoted $\tilde{\delta n_{j}}$, and the electric field defined on the grid points $\tilde{E}_{j}$.

\subsection{The Shape Function}

The shape function $S$ is used to interpolate between discrete and continuum quantities in the simulation model. To perform the spatial grid analysis, we relate the Fourier representations of $\tilde{\delta n}$ to $\delta n^{(1)}$ and $E^{(1)}$ to $\tilde{E}$. As in [2], we have

$$
\tilde{\delta n}(k)=\sum_{q} S\left(k_{q}\right) \delta n^{(1)}\left(k_{q}\right)
$$

where $k_{q}=k-2 \pi q / \Delta x$ with the summation over all $q \in \mathbb{Z}$. The presence of $S(k)$ accounts for the finite size of the computational particles, and the summation over spatial aliases accounts for the discrete sampling on the grid points. For the electric field, we have

$$
E^{(1)}(k)=S(-k) \tilde{E}(k),
$$

where the presence of $S(k)$ accounts for the interpolation to the locations of the particles.

The shape function is typically taken to be a B-Spline function. For illustration, we consider a linear B-Spline for our model given explicitly by

$$
\Delta x S(x)= \begin{cases}1-\frac{|x|}{\Delta x} & :|x| \leq \Delta x \\ 0 & :|x|>\Delta x .\end{cases}
$$

The FS coefficients of $S$ are given by

$$
S(k)=\operatorname{dif}^{2}\left(\frac{k \Delta x}{2}\right), \quad \operatorname{dif}(\theta) \equiv \frac{\sin \theta}{\theta}
$$

for each allowed value of $k$.

\subsection{Modified Dispersion Relations}

We now use the spatial grid analysis outlined above to derive modified dispersion relations for the $\delta f$ and full- $f$ methods to include the effects of $\Delta t$ and $\Delta x$. We first illustrate the technique with the $\delta f$ method and then simply present the result for the full- $f$ method. Beginning with Eq.(34), we plug into Eq. 47) 
and replace $E^{(1)}$ with $\tilde{E}$ using Eq. (48). Since $\tilde{E}$ is periodic in Fourier space, it can be pulled out of the summation, yielding

$$
\tilde{\delta n}(k, z)=\frac{e}{m_{i}} \tilde{E}(k, z) \sum_{q=-\infty}^{\infty} S\left(k_{q}\right) S\left(-k_{q}\right) \int_{-\infty}^{\infty}\left[\frac{\Delta t}{1-z e^{i k_{q} v \Delta t}}\right] \frac{\partial f_{0}}{\partial v} d v .
$$

Next, we use a discrete version of our field model, Eq. 21), to relate $\tilde{\delta n}$ and $\tilde{E}$. We consider a discrete spatial model in which derivatives are taken spectrally, so the DFT version of Eq. 21] is simply

$$
\tilde{E}(k, z)=-i k \frac{T_{e}}{e n_{0}} \tilde{\delta n}(k, z) .
$$

In this case, the spatial derivative corresponds to a multiplication by $i k$ in the DFT. If another method is used to compute the spatial derivative, this would need to be accounted for in the analysis. For example, a spatial derivative calculated from a centered finite difference scheme would correspond to a multiplication by $i k \operatorname{dif}(k \Delta x)$ in the DFT [2].

Using Eq.50], Eq.(51), and Eq.(52), we arrive at the modified dispersion relation for the $\delta f$ method

$$
\epsilon_{\delta f}(k, z) \equiv 1+\frac{k T_{e}}{m_{i} n_{0}} \sum_{q=-\infty}^{\infty} \operatorname{dif}^{4}\left(\frac{k_{q} \Delta x}{2}\right) \int_{-\infty}^{\infty}\left[\frac{i \Delta t}{1-z e^{i k_{q} v \Delta t}}\right] \frac{\partial f_{0}}{\partial v} d v=0,
$$

which for $f_{0}=f_{M}$ gives

$$
\epsilon_{\delta f}(\zeta)=1-\frac{1}{2 T} \sum_{q=-\infty}^{\infty} \operatorname{dif}^{4}\left(\frac{k_{q} \Delta x}{2}\right) X_{\delta f}\left(\frac{\zeta}{\sqrt{2}} ; \sqrt{2} k v_{t h} \Delta t, \frac{2 \pi q}{k \Delta x}\right)=0 .
$$

with $T$ and $\zeta$ defined as in Eq. 24 . Repeating the above process using the full- $f$ linear perturbed number density, Eq. (40), gives the modified dispersion relation

$$
\epsilon_{f}(k, z) \equiv 1+\frac{k T_{e}}{m_{i} n_{0}} \sum_{q=-\infty}^{\infty} \operatorname{dif}^{4}\left(\frac{k_{q} \Delta x}{2}\right) k_{q} \int_{-\infty}^{\infty}\left[\frac{\Delta t}{1-z e^{i k_{q} v \Delta t}}-\alpha \Delta t\right]^{2} f_{0} d v=0,
$$

which for $f_{0}=f_{M}$ gives

$$
\epsilon_{f}(\zeta)=1-\frac{1}{2 T} \sum_{q=-\infty}^{\infty} \operatorname{dif}^{4}\left(\frac{k_{q} \Delta x}{2}\right) X_{f}\left(\frac{\zeta}{\sqrt{2}} ; \sqrt{2} k v_{t h} \Delta t, \frac{2 \pi q}{k \Delta x}, \alpha\right)=0 .
$$

\section{Analysis of the Modified Dispersion Relations}

In this section, we analyze the modified dispersion relations Eq.(53), Eq. (54), Eq. (55), and Eq. (56) to obtain results regarding the numerical accuracy and stability of the $\delta f$ and full- $f$ PIC methods. We study the accuracy of the real frequency and damping rate using a perturbation method applied to the dispersion relations with Maxwellian equilibrium distributions Eq.(54) and Eq.(56). The stability of the methods is examined analytically for a cold ion plasma and numerically for a finite temperature Maxwellian plasma.

\subsection{Dispersion Accuracy}

To study the accuracy of the real frequency and damping rate in a Maxwellian plasma for our PIC methods, we compare the scaled phase velocities $\zeta$ obtained from Eq.(54) or Eq. (56) to the Vlasov result $\zeta_{0}$ obtained from Eq. 224. We assume that $\zeta$ has a regular double perturbation series in the small parameters $\epsilon_{1}=k v_{t h} \Delta t$ and $\epsilon_{2}=k \Delta x$ given as

$$
\zeta=\zeta_{0}+\epsilon_{1} \zeta_{(1,0)}+\epsilon_{2} \zeta_{(0,1)}+\epsilon_{1}^{2} \zeta_{(2,0)}+\epsilon_{1} \epsilon_{2} \zeta_{(1,1)}+\epsilon_{2}^{2} \zeta_{(0,2)}+\ldots
$$


This form can be plugged into Eq. (54) or Eq. (56). The modified dispersion relation is then expanded in a double Taylor series in $\epsilon_{1}$ and $\epsilon_{2}$ and powers of $\epsilon_{1}^{n} \epsilon_{2}^{m}$ are collected. Performing this procedure with Eq. 54 yields for the $\delta f$ method $\zeta_{(1,0)}=\zeta_{(0,1)}=\zeta_{(1,1)}=0$, giving second order correction terms of

$$
\zeta-\zeta_{0} \sim \frac{\sqrt{2}}{6 Z^{\prime \prime}\left(\zeta_{0} / \sqrt{2}\right)}\left[2 T(k \Delta x)^{2}-\left(k v_{t h} \Delta t\right)^{2}\right] .
$$

We note that the Vlasov theory yields a non-dispersive ion acoustic wave, but numerical dispersion is introduced in the discrete model from $\Delta t$ and $\Delta x$. An examination of the signs of the real and imaginary parts for the terms on the right hand side shows the finite time step will act to increase the frequency and weaken the damping. The finite spatial step will have the opposite effect. Taking the ratio $v_{t h} \Delta t / \Delta x=\sqrt{2 T}$ will balance the effects and result in a higher order of accuracy.

We validate the expression Eq.(58) by performing linear $\delta f$ simulations varying the size of $k v_{t h} \Delta t$ for a fixed value of $k \Delta x=\pi / 8$. The relative difference in the real frequency, $\omega_{R}=\operatorname{Re}(\omega)$, and damping rate, $\gamma=\operatorname{Im}(\omega)$, between the values predicted in the Vlasov theory and those produced in simulations are measured and plotted in figure 1 . In the simulations, we choose $T=0.1$ which provides a low damping rate, so measurements can be made accurately. A large number of computational particles $\left(N_{p}=2097152\right)$ are used to make the errors due to the finite number of particles small compared to the error due to $\Delta t \neq 0$ and $\Delta x \neq 0$. To further reduce errors associated with discrete particle effects, we use the Hammersley set (bit-reversed numbers) [13 20] in the particle loading process. This allows us to simulate Landau-damped ion acoustic modes for long periods of time and obtain accurate measurements of $\omega_{R}$ and $\gamma$. The simulation error results are in excellent agreement with the error predicted from numerically solving Eq. (54) and taking the difference with the solution of Eq.(24). We refer to this difference as the theoretical numerical error. The asymptotic result Eq. (58) is also shown to be valid for small $k v_{t h} \Delta t$. Finally, the simulation results also confirm that the numerical dispersion is independent of $\alpha$ for the $\delta f$ method.

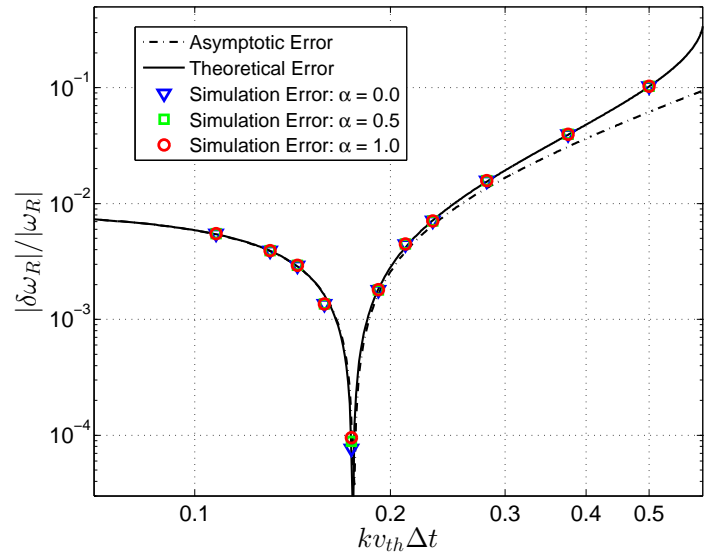

(a) Relative error in real frequency

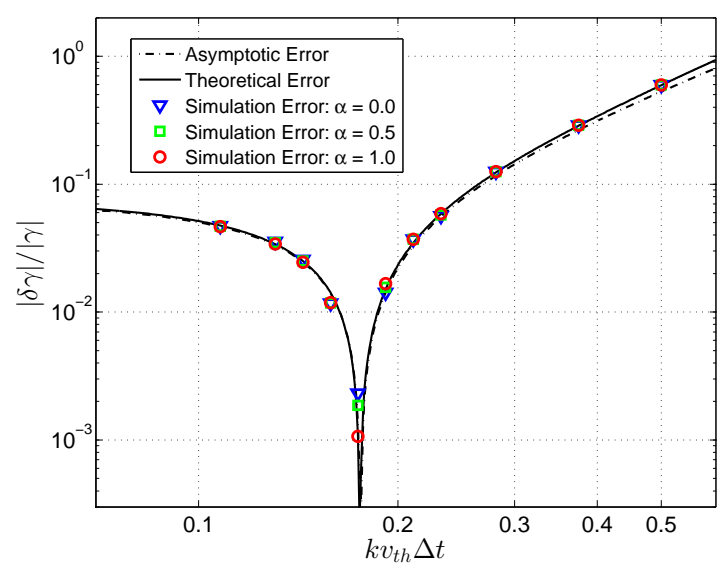

(b) Relative error in damping rate

Figure 1: Simulation results varying the size of $k v_{t h} \Delta t$ for the $\delta f$ method are compared with the theoretical numerical error and the asymptotic error for values of $\alpha=0,1 / 2$, and 1 . It can be seen that errors decrease rapidly near the region where $v_{t h} \Delta t / \Delta x=\sqrt{2 T}$. As $k v_{t h} \Delta t$ continues to decrease past this region, the error is due to finite $k \Delta x$.

Repeating this analysis for the full- $f$ method using Eq. (56), we find the correction to $\zeta$ at the lowest order to be

$$
\zeta-\zeta_{0} \sim \frac{\sqrt{2}}{6 Z^{\prime \prime}\left(\zeta_{0} / \sqrt{2}\right)}\left[\frac{12 i(2 \alpha-1)}{\zeta_{0}}(T+1)\left(k v_{t h} \Delta t\right)+2 T(k \Delta x)^{2}\right]
$$

showing the method to produce results which are first order accurate in time for all $\alpha$ except $\alpha=1 / 2$. The 
effect of $\Delta x$ is again to lower the frequency and strengthen the damping. The effect of $\Delta t$ is more difficult to analyze directly from Eq. $(59)$ and is dependent on both $T$ and $\alpha$. For $\alpha=1 / 2$, the full- $f$ method will produce results which are second order accurate in time. The correction to $\zeta$ is then given by

$$
\zeta-\zeta_{0} \sim \frac{\sqrt{2}}{3 Z^{\prime \prime}\left(\zeta_{0} / \sqrt{2}\right)}\left[\left(k v_{t h} \Delta t\right)^{2}+T(k \Delta x)^{2}\right] .
$$

Again, we compare the results of Eq.(59) and Eq. (60) with simulations varying the size of $k v_{t h} \Delta t$. The same values for $N_{p}$ and $k \Delta x$ are used as with the $\delta f$ method. These results are presented in figure 2.

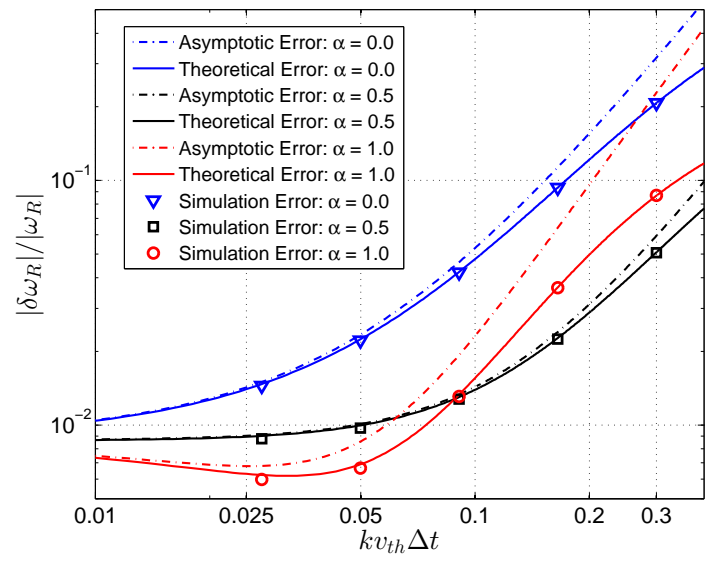

(a) Relative error in real frequency

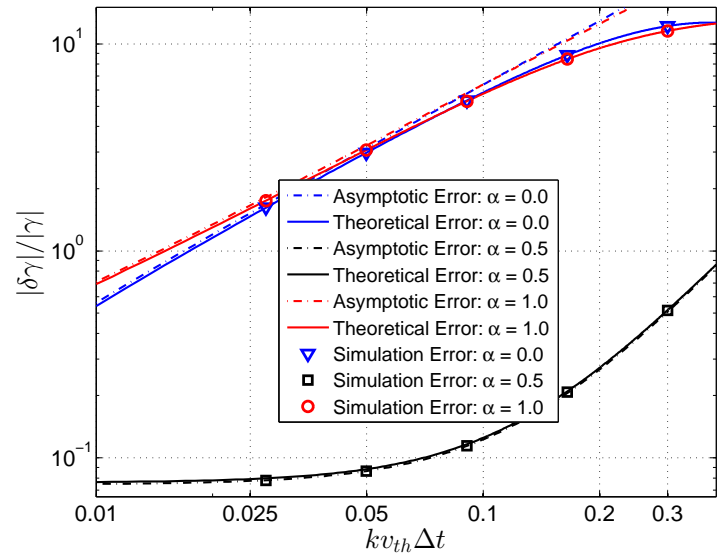

(b) Relative error in damping rate

Figure 2: Simulation results varying the size of $k v_{t h} \Delta t$ for the full- $f$ method are compared with the theoretical numerical error and the asymptotic error, including second order terms, for values of $\alpha=0,1 / 2$, and 1 .

Finally, we test the convergence of the $\delta f$ and full- $f$ methods in $k \Delta x$, fixing $k v_{t h} \Delta t=0.075$ and $\alpha=1 / 2$. The results are presented in figure 3 .

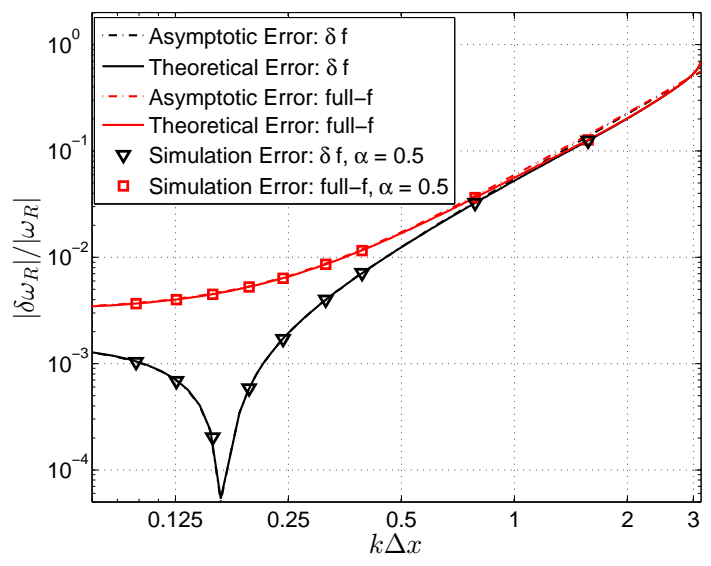

(a) Relative error in real frequency

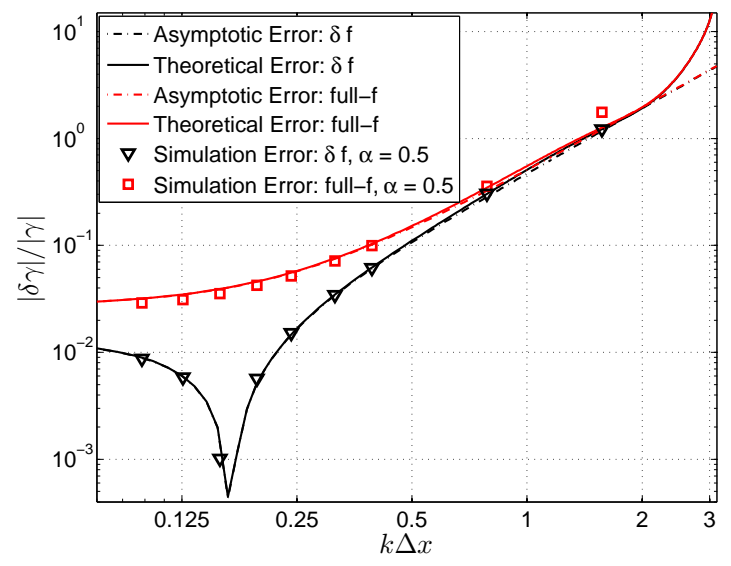

(b) Relative error in damping rate

Figure 3: Simulation results varying the size of $k \Delta x$ for both the $\delta f$ and full- $f$ methods are compared with the theoretical numerical error and the asymptotic error. 


\subsection{Numerical Stability Analysis for Cold Ions}

Next, we consider how the full- $f$ and $\delta f$ PIC methods behave in the cold ion limit, which allows for a straight-forward analysis of the modified dispersion relations. In the next section, we will examine the stability properties with increasing ion temperature. For the cold ion stability analysis, we begin with the $\delta f$ method, choosing $f_{0}(v)=n_{0} \delta(v)$ in Eq. 53). With this choice, the velocity integration and the summation can be performed exactly, and we are left with a quadratic equation for the amplification factor $z$ given by

$$
(1-z)^{2}+z\left(k c_{s} \Delta t\right)^{2} \operatorname{dif}\left(\frac{k \Delta x}{2}\right) \cos \left(\frac{k \Delta x}{2}\right)=0,
$$

where $c_{s} \equiv \sqrt{T_{e} / m_{i}}$ is the ion sound speed. The solution to Eq. 61) gives stability restrictions on $k \Delta x$ and $k c_{s} \Delta t$ from the requirement that $|z| \leq 1$. The region in this parameter space that will produce stable solutions is shown in figure 4. A simple condition to ensure stability at any wave number can be given by

$$
c_{s} \frac{\Delta t}{\Delta x} \leq 1.483
$$

which is obtained by finding the maximum possible slope of a line segment through parameter space connecting the points $(0,0)$ and $\left(\pi, k c_{s} \Delta t\right)$ and contained entirely in the region of linear stability. This is in a form similar to a Courant-Friedrichs-Lewy (CFL) condition 21, which is not typically observed when using implicit time integration schemes.

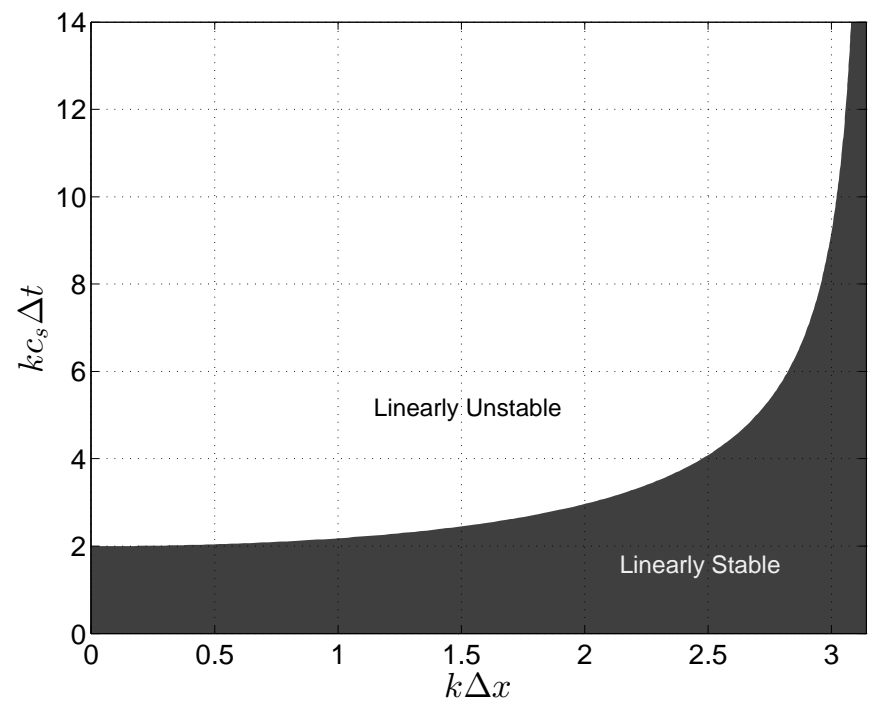

Figure 4: The cold ion stability region for the $\delta f$ method is plotted. The shaded area represents regions in the parameter space $\left(k \Delta x, k c_{s} \Delta t\right)$ which will result in stable simulations.

For the full- $f$ method, choosing $f_{0}(v)=n_{0} \delta(v)$ in Eq. 55 gives the following equation for the amplification factor

$$
(1-z)^{2}+(1-\alpha+\alpha z)^{2}\left(k c_{s} \Delta t\right)^{2} \operatorname{dif}\left(\frac{k \Delta x}{2}\right) \cos \left(\frac{k \Delta x}{2}\right)=0 .
$$

Solving this equation gives for the square modulus of either root

$$
|z|^{2}=\frac{k \Delta x+\left(k c_{s} \Delta t\right)^{2}(1-\alpha)^{2} \sin (k \Delta x)}{k \Delta x+\left(k c_{s} \Delta t\right)^{2} \alpha^{2} \sin (k \Delta x)},
$$


which is greater than unity when $\alpha<1 / 2$ and less than unity when $\alpha>1 / 2$, independent of $k c_{s} \Delta t$ and $k \Delta x$. Hence the cold ion stability of the full- $f$ method is determined by the implicitness of the scheme, as is usually expected, and no CFL condition is present.

\subsection{Numerical Stability Analysis for Warm Ions}

A stability analysis for the warm Maxwellian equilibrium distribution is more difficult, requiring the numerical solutions of Eq.(54) and Eq.(56). A discussion on the numerical solution of integrals of similar form to Eq.(37) and Eq.(42) can be found in the appendix of [1. A search for the boundary of the stability region is performed by numerically solving the dispersion relations over a region of the parameter space $\left(k \Delta x, k v_{t h} \Delta t\right)$ and determining the contours for which $|z|=1$. Stability conditions of the form

$$
r \equiv v_{t h} \frac{\Delta t}{\Delta x} \leq C_{\max }
$$

are then determined from the stability region boundaries to ensure stability at all present wave numbers. Here, $C_{\max }$ is the Courant number [21, which depends on $T$ for warm ions.

\subsubsection{Warm Ion Stability for the $\delta f$ Method}

The stability region boundaries for the $\delta f$ method are shown in figure 5 for various values of $T$. It is observed that the $\delta f$ method shows better stability as $T$ is increased. The unstable regions become smaller for higher temperatures and vanish altogether for temperatures above a critical value between $T=0.6$ and $T=0.7$.

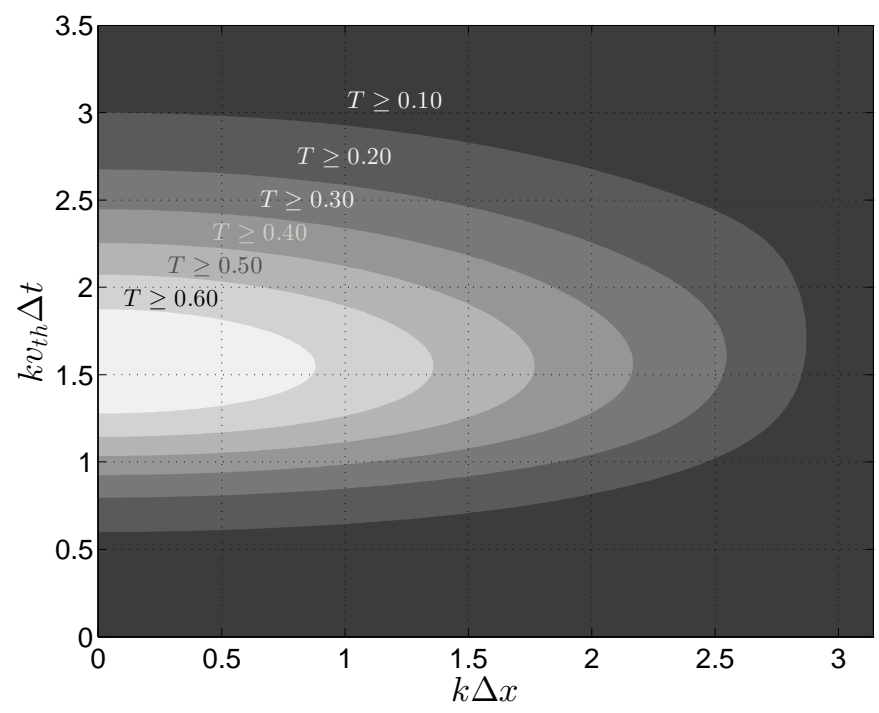

Figure 5: Stability regions for the $\delta f$ method are plotted for various values of $T$. The different shaded areas represent regions in the parameter space $\left(k \Delta x, k v_{t h} \Delta t\right)$ which will result in stable simulations for $T$ above the indicated value.

Courant numbers are given in table 1 for the different values of $T$, along with the first mode to become unstable as $r$ is increased past $C_{\max }$. Finally, a predicted instability for $T=0.3$ is demonstrated by performing the simulations shown in figures 6 and 7 . The initial conditions are taken such that each nonzero wave number in the DFT begins with a finite perturbation, as discussed in Appendix B. The simulation in figure 6 is observed to be stable at all wave numbers with $r=0.62$. In figure $7, r=0.66$ and the modes near $k \Delta x=1.96$ are shown to exhibit exponential growth. This is in agreement with the predicted instability for $r>0.64$. It is observed from the numerical solution of Eq. (54) and simulations that $\omega_{R} \Delta t=\pi$ 
at the onset of the instability in the $\delta f$ method. This is thought to be due to inadequate temporal resolution of the ion acoustic wave for the first unstable DFT wave number, since the physical wave from Eq. 24. has a real frequency exceeding $\pi / \Delta t$.

\begin{tabular}{|c|c|c|}
\hline$T$ & $C_{\max }$ & $k \Delta x$ \\
\hline 0.10 & 0.40 & 2.27 \\
0.20 & 0.51 & 2.19 \\
0.30 & 0.64 & 1.96 \\
0.40 & 0.82 & 1.66 \\
0.50 & 1.09 & 1.31 \\
0.60 & 1.73 & 0.87 \\
0.70 & $\infty$ & - \\
\hline
\end{tabular}

Table 1: Courant numbers $\left(C_{\max }\right)$ are given for warm $\delta f$ ions for various values of $T$, along with the first discrete mode to become unstable $(k \Delta x)$ as $r$ is increased past $C_{\max }$.

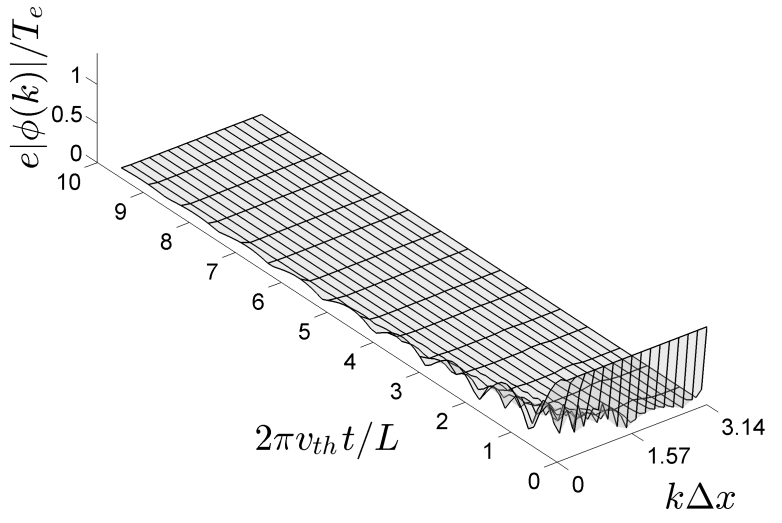

(a) Time history of DFT modes of $e \phi / T_{e}$

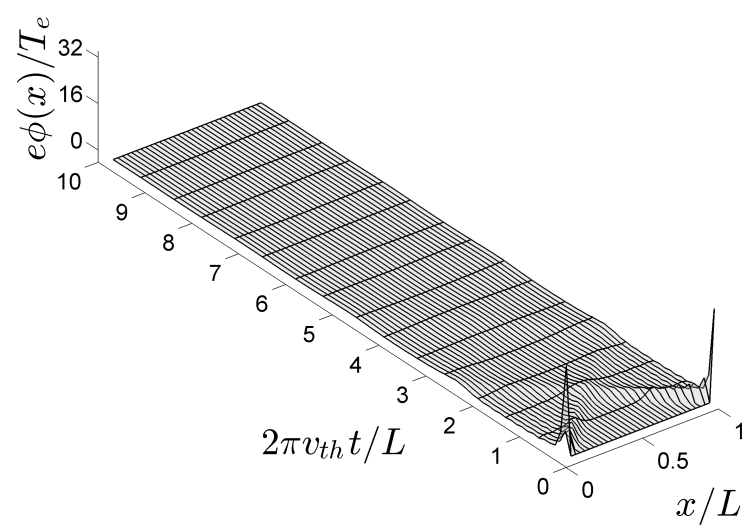

(b) Time history of $e \phi / T_{e}$ in real space

Figure 6: $\delta f$ simulation for $T=0.3$ and $r=0.62$. The initial condition is taken to give a finite perturbation to each non-zero wave number in the DFT. The simulation is stable at all wave numbers, in agreement with the theory which predicts stability for $r<0.64$.

\subsubsection{Warm Ion Stability for the Full-f Method}

For the full- $f$ method, it is observed that unconditional stability is maintained for $\alpha \geq 0.5$ as temperature is increased. For $\alpha<0.5$, simulations are no longer unconditionally unstable, but instead develop stability regions in $\left(k \Delta x, k v_{t h} \Delta t\right)$ space. Again stability is improved as $T$ is increased, and for fixed values of $\alpha<0.5$, there are critical temperatures for which the unstable regions vanish making the method unconditionally stable. An example of the finite temperature stability regions is shown in figure 8 for $\alpha=0.250$. The critical temperature occurs between $T=0.18$ and $T=0.20$.

Courant numbers are given in table 2 for $\alpha=0.125,0.250$, and 0.375 at different values of $T$. Finally, a predicted instability for $\alpha=0.250$ at $T=0.14$ is demonstrated by performing the simulations shown in figures 9 and 10. The initial conditions are again taken to excite each non-zero wave number in the DFT with a finite perturbation. The simulation in figure 9 is observed to be stable for $r=0.16$. In figure 10 , $r=0.24$ and the modes near $k \Delta x=0.91$ are shown to grow with time. This is in agreement with the predicted instability for $r>0.20$. It is observed that the instabilities for the full- $f$ method occur for values 


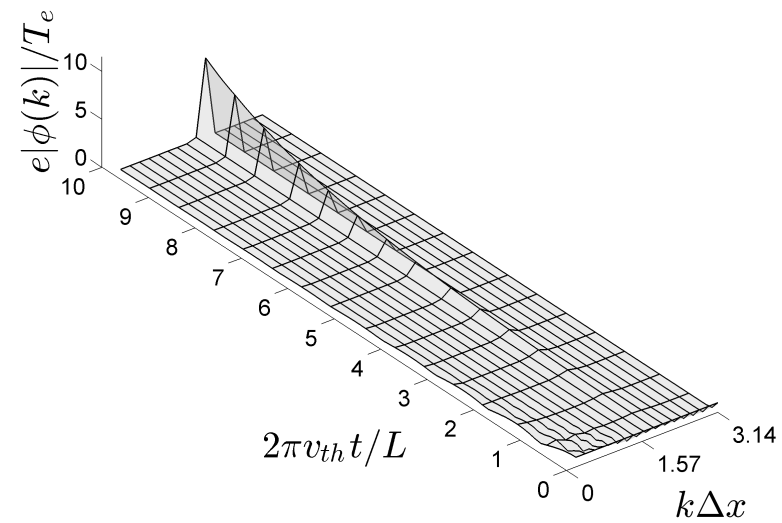

Figure 7: $\delta f$ simulation for $T=0.3$ and $r=0.66$. The time history of the DFT modes is shown with the initial condition taken to give a finite perturbation to each non-zero wave number in the DFT. Exponential growth of the modes near $k \Delta x=1.96$ is observed for $r=0.66$, in agreement with the predicted instability for $r>0.64$.

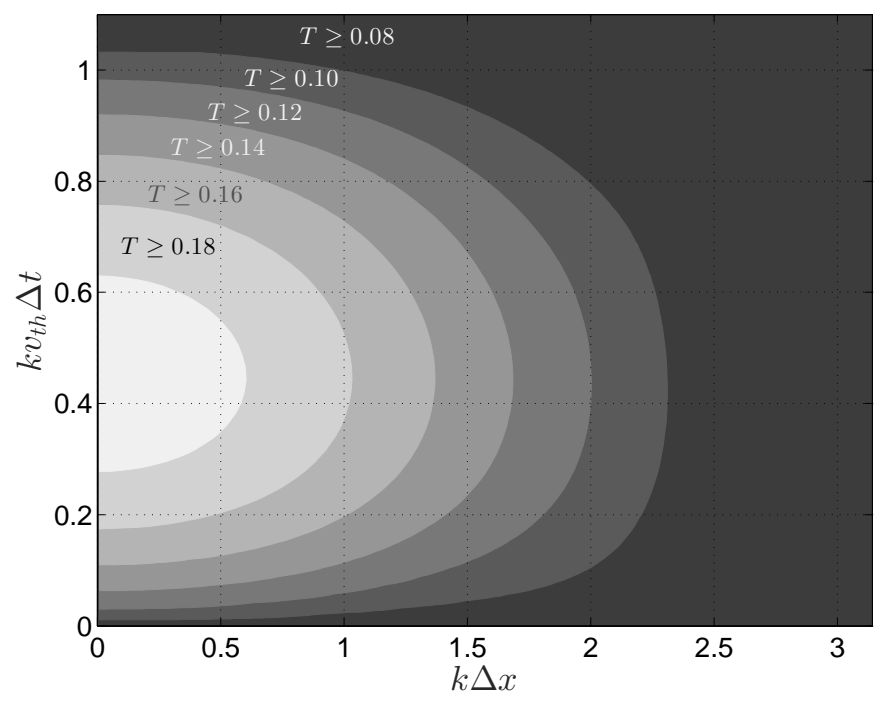

Figure 8: Stability regions for the full- $f$ method with $\alpha=0.25$ are plotted for various values of $T$. The different shaded areas represent regions in the parameter space $\left(k \Delta x, k v_{t h} \Delta t\right)$ which will result in stable simulations for $T$ above the indicated value.

$\omega_{R} \Delta t<\pi$. The physical wave from Eq. (24) can be resolved on the time "grid" for the first unstable DFT wave number in this case, having a real frequency less than $\pi / \Delta t$.

\section{Summary}

In this paper, we have developed a theoretical framework for analyzing implicit and explicit time integration schemes applied to $\delta f$ method PIC models. This work is easily combined with the spatial grid analysis of Langdon to provide modified dispersion relations to include the numerical effects of both $\Delta t$ and $\Delta x$. The analysis is illustrated for the adjustable time centering implicit scheme [8. A significant challenge is to 
$\alpha=0.125$

\begin{tabular}{|c|c|c|}
\hline$T$ & $C_{\max }$ & $k \Delta x$ \\
\hline 0.08 & 0.02 & 0.85 \\
0.10 & 0.04 & 0.92 \\
0.12 & 0.07 & 0.97 \\
0.14 & 0.12 & 0.98 \\
0.16 & 0.19 & 0.95 \\
0.18 & 0.28 & 0.88 \\
0.20 & 0.45 & 0.74 \\
0.22 & 1.05 & 0.40 \\
0.24 & $\infty$ & - \\
\hline
\end{tabular}

$\alpha=0.250$

\begin{tabular}{|c|c|c|}
\hline$T$ & $C_{\max }$ & $k \Delta x$ \\
\hline 0.08 & 0.02 & 0.84 \\
0.10 & 0.06 & 0.91 \\
0.12 & 0.11 & 0.94 \\
0.14 & 0.20 & 0.91 \\
0.16 & 0.33 & 0.82 \\
0.18 & 0.68 & 0.56 \\
0.20 & $\infty$ & - \\
0.22 & $\infty$ & - \\
0.24 & $\infty$ & - \\
\hline
\end{tabular}

$\alpha=0.375$

\begin{tabular}{|c|c|c|}
\hline$T$ & $C_{\max }$ & $k \Delta x$ \\
\hline 0.08 & 0.05 & 0.83 \\
0.10 & 0.13 & 0.86 \\
0.12 & 0.28 & 0.77 \\
0.14 & 1.48 & 0.25 \\
0.16 & $\infty$ & - \\
0.18 & $\infty$ & - \\
0.20 & $\infty$ & - \\
0.22 & $\infty$ & - \\
0.24 & $\infty$ & - \\
\hline
\end{tabular}

Table 2: Courant numbers $\left(C_{\max }\right)$ are given for warm full- $f$ ions for various values of $T$ and $\alpha<1 / 2$, along with the first discrete mode to become unstable $(k \Delta x)$ as $r$ is increased past $C_{\max }$.

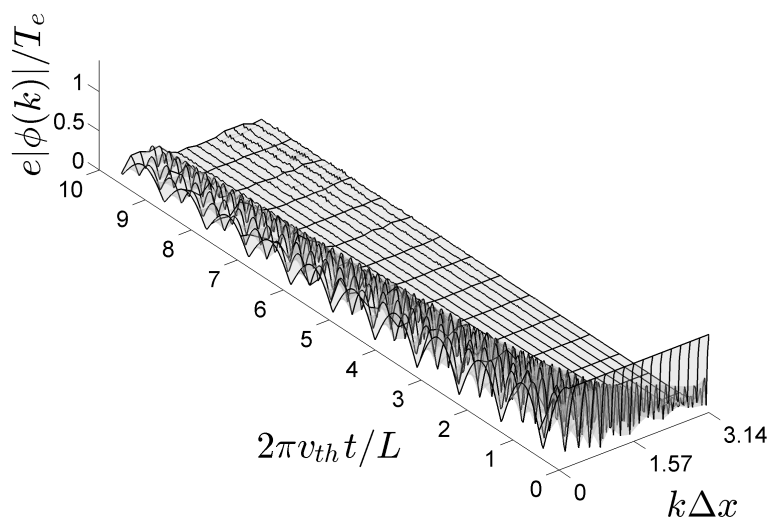

(a) Time history of DFT modes of $e \phi / T_{e}$

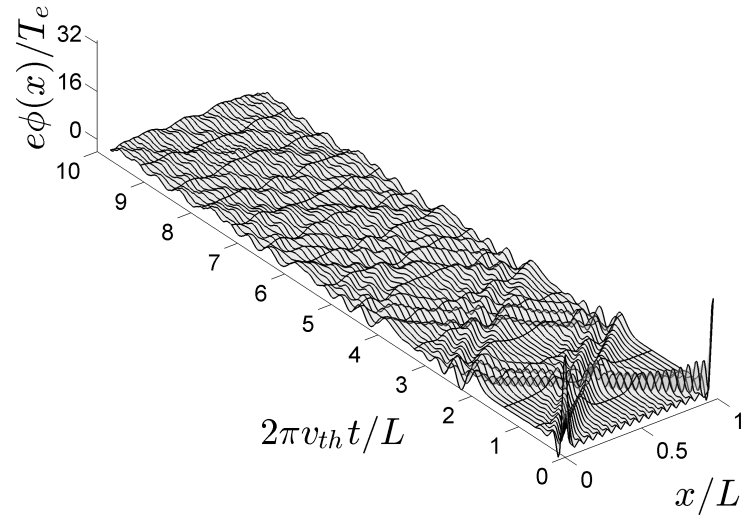

(b) Time history of $e \phi / T_{e}$ in real space

Figure 9: Full- $f$ simulation for $T=0.14, \alpha=0.25$, and $r=0.16$. The initial condition is taken to give a finite perturbation to each non-zero wave number in the DFT. The simulation is stable at all wave numbers, in agreement with the theory which predicts stability for $r<0.20$.

analyze the resulting modified dispersion relations that include finite $\Delta t$ and $\Delta x$. We used a perturbative method to obtain lowest order corrections to the real frequency and damping rate due to $\Delta t$ and $\Delta x$. This provides a theory for the accuracy of the simulations and can also provide some insights on the stability of various $\delta f$ schemes. Further exploring the issue of numerical stability, we find that a CFL condition exists in the $\delta f$ method PIC model at low ion temperatures, independent of the parameter $\alpha$, which characterizes the implicitness of the scheme. We note that this unusual behavior occurs only for implicit $\delta f$ PIC with low temperature ions. Full- $f$ PIC does not exhibit this behavior and can be made unconditionally stable with sufficient implicitness. For cold ions, this condition can become restrictive for increasing grid resolution. The CFL condition for this particular model illustrates an unusual issue that may be encountered when using the $\delta f$ method. In particular, it is possible for implicit schemes to provide no additional stability. 


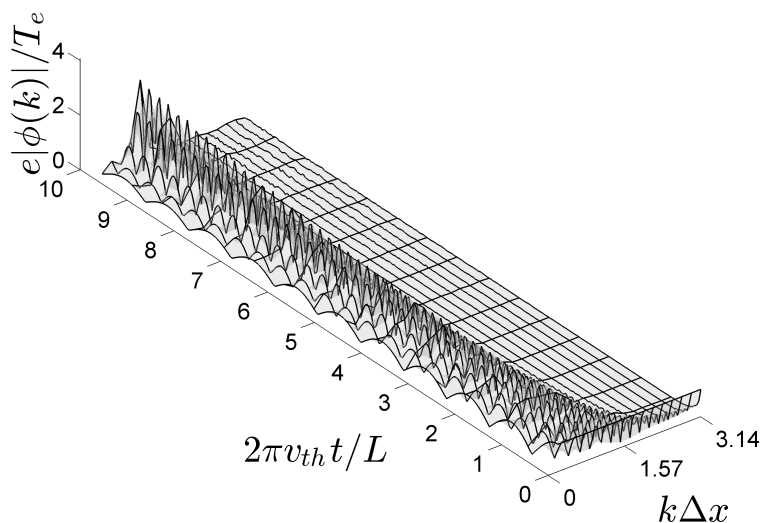

Figure 10: Full- $f$ simulation for $T=0.14, \alpha=0.25$, and $r=0.24$. The time history of the DFT modes is shown with the initial condition taken to give a finite perturbation to each non-zero wave number in the DFT. Exponential growth of the modes near $k \Delta x=0.91$ is observed for $r=0.24$, in agreement with the predicted instability for $r>0.20$.

\section{Appendix A. Linearization of the $\delta f$ Method}

To linearize the $\delta f$ method, we let $\epsilon$ be a parameter representing the perturbation size at $t=0$. For example, $\epsilon$ may be present in an initial condition as

$$
w_{p}(t=0)=\epsilon g\left(\mathbf{x}_{p}(t=0), \mathbf{v}_{p}(t=0)\right) .
$$

where $g(\mathbf{x}, \mathbf{v}) \sim O(1)$. We assume that all quantities in the model are analytic in $\epsilon$ around the unperturbed system and denote the $\epsilon$ dependence of a time dependent quantity by $\psi(t ; \epsilon)$. The unperturbed system, corresponding to $\epsilon=0$, is given by

$$
\mathbf{E}_{j}(t ; 0)=\mathbf{0}, \quad \delta n_{j}(t ; 0)=0, \quad w_{p}(t ; 0)=0, \quad \mathbf{v}_{p}(t ; 0)=\mathbf{v}_{p}^{(0)}, \quad \mathbf{x}_{p}(t ; 0)=\mathbf{x}_{p}^{(0)}(t)
$$

This allows an expansion in $\epsilon$ as

$$
\begin{aligned}
\mathbf{E}_{j}(t ; \epsilon) & =\epsilon \mathbf{E}_{j}^{(1)}(t)+\ldots \\
\delta n_{j}(t ; \epsilon) & =\epsilon \delta n_{j}^{(1)}(t)+\ldots \\
w_{p}(t ; \epsilon) & =\epsilon w_{p}^{(1)}(t)+\ldots \\
\mathbf{v}_{p}(t ; \epsilon) & =\mathbf{v}_{p}^{(0)}+\epsilon \mathbf{v}_{p}^{(1)}(t)+\ldots \\
\mathbf{x}_{p}(t ; \epsilon) & =\mathbf{x}_{p}^{(0)}(t)+\epsilon \mathbf{x}_{p}^{(1)}(t)+\ldots .
\end{aligned}
$$

We note that for a quantity $\psi(t ; \epsilon)$ to be accurately approximated as

$$
\psi(t ; \epsilon) \approx \psi^{(0)}(t)+\epsilon \psi^{(1)}(t),
$$

it is necessary that $\epsilon^{m} \psi^{(m)}(t) \ll \psi^{(0)}(t)+\epsilon \psi^{(1)}(t)$ for all $m \geq 2$. This requirement, however, is not guaranteed to hold uniformly in time for the linearized model. The linearized model, therefore, should only be expected to accurately model the behavior of the nonlinear system on short time scales after a small perturbation. 
Proceeding with the expansion in Eq. A.3), we compute the perturbed number density at grid point $\mathbf{X}_{j}$ from Eq. 10, including terms to $O(\epsilon)$. We have

$$
\delta n_{j}(t ; \epsilon)=\sum_{p} w_{p}(t ; \epsilon) S\left(\mathbf{X}_{j}-\mathbf{x}_{p}(t ; \epsilon)\right)=\epsilon \sum_{p} w_{p}^{(1)}(t) S\left(\mathbf{X}_{j}-\mathbf{x}_{p}^{(0)}(t)\right)+O\left(\epsilon^{2}\right),
$$

yielding

$$
\delta n_{j}^{(1)}(t)=\sum_{p} w_{p}^{(1)}(t) S\left(\mathbf{X}_{j}-\mathbf{x}_{p}^{(0)}(t)\right) .
$$

77 Next, to compute $w_{p}^{(1)}(t)$, we plug Eq. A.3 into the nonlinear particle weight equation Eq. 8. and expand to $O(\epsilon)$. This gives

$$
\frac{d w_{p}^{(1)}(t)}{d t}=-\left.\frac{e}{m_{i}} \mathbf{E}^{(1)}\left(\mathbf{x}_{p}^{(0)}(t), t\right) \cdot \nabla_{\mathbf{v}} \ln f_{0}(\mathbf{v})\right|_{\mathbf{v}_{p}^{(0)}},
$$

379

where $\mathbf{E}^{(1)}\left(\mathbf{x}_{p}^{(0)}(t), t\right)$ is the $O(\epsilon)$ term of the interpolated electric field

$$
\mathbf{E}^{(1)}\left(\mathbf{x}_{p}^{(0)}(t), t\right)=\Delta x \sum_{j} \mathbf{E}_{j}^{(1)}(t) S\left(\mathbf{X}_{j}-\mathbf{x}_{p}^{(0)}(t)\right) .
$$

We note that Eq. A.6] and Eq. A.7] require only the computation of the unperturbed particle orbits. This is in contrast to the linearization of the full- $f$ method, which requires the first order perturbations of the particle orbits. Plugging Eq. A.3 into Eq. 2], we have for the unperturbed particle orbits, simply,

$$
\begin{aligned}
\frac{d \mathbf{x}_{p}^{(0)}(t)}{d t} & =\mathbf{v}_{p}^{(0)} \\
\frac{d \mathbf{v}_{p}^{(0)}}{d t} & =0 .
\end{aligned}
$$

Finally, for the first order electric field at grid point $\mathbf{X}_{j}$, we plug Eq. A.3 into a discretized version of Eq. 21). This yields

$$
\mathbf{E}_{j}^{(1)}(t)=\frac{T_{e}}{e n_{0}} \mathbf{D} \delta n_{j}^{(1)}(t),
$$

where $\mathbf{D}$ is a discrete gradient operator.

\section{Appendix B. Initialization}

In our tests of numerical stability, an initial condition of the form

$$
f(x, v, t=0)=\left(1+\frac{\delta n^{(1)}(x, t=0)}{n_{0}}\right) f_{0}(v)
$$

is used for both the $\delta f$ and full- $f$ PIC simulations, where $\delta n^{(1)}(x, t=0)$ is chosen to excite each non-zero wave number in the DFT with a finite perturbation. To initialize the perturbed density such that the DFT is approximately constant across the non-zero wave numbers, the filtering due to the shape function must be accounted for. To counter this effect, a Fourier filter $H(k)$ is used with the FS of the initial condition. The filter defined by:

$$
H(k)=\left\{\begin{array}{lll}
\frac{1}{S(k)} & : & k \in\left(-\frac{\pi}{\Delta x}, \frac{\pi}{\Delta x}\right] \backslash\{0\} \\
0 & : \quad k \in\left(-\infty,-\frac{\pi}{\Delta x}\right] \cup\{0\} \cup\left(\frac{\pi}{\Delta x}, \infty\right)
\end{array}\right.
$$


where $A$ is the amplitude at which we wish to excite the DFT modes. Notice from Eq.(B.1), we have

$$
\frac{\delta f(x, v, t=0)}{f_{0}(v)}=\frac{\delta n^{(1)}(x, t=0)}{n_{0}} .
$$

where

$$
P^{(1)}(x, t=0)=\int_{-\infty}^{\infty} x^{(1)}(x, v, t=0) f_{0}(v) d v .
$$

By choosing $x^{(1)}$ to be independent of $v$ at $t=0$, this is simply

$$
P^{(1)}(x, t=0)=n_{0} x^{(1)}(x, t=0) .
$$

and

$$
x^{(1)}(x, t=0)=A \operatorname{Re}\left\{\sum_{k \neq 0} \frac{i H(k)}{k} e^{i k x}\right\},
$$

and the initial particle velocity perturbation is taken as zero. To implement this initial condition then, the unperturbed particle positions $x_{p}^{(0)}$ are loaded uniformly in space, and the perturbed particle data is initialized by

$$
x_{p}^{(1)}(t=0)=A \operatorname{Re}\left\{\sum_{k \neq 0} \frac{i H(k)}{k} e^{i k x_{p}^{(0)}(t=0)}\right\}
$$

$$
v_{p}^{(1)}(t=0)=0 .
$$

\section{Acknowledgements}

We would like to thank Drs. Y. Chen and T. Manteuffel for their helpful discussions. This work was supported by the U.S. Department of Energy grant DE-FG02-08ER54954. 
[1] A. B. Langdon, J. Comput. Phys. 30 (1979) 202.

[2] A. B. Langdon, J. Comput. Phys. 6 (1970) 247.

[3] S. E. Parker, W. W. Lee, Phys. Fluids B 5 (1993) 77.

[4] A. M. Dimits, W. W. Lee, J. Comput. Phys. 107 (1993) 309.

[5] G. Hu, J. A. Krommes, Phys. Plasmas 1 (1994) 863.

[6] A. Y. Aydemir, Phys. Plasmas 1 (1994) 822.

[7] M. Kotschenreuther, Bull. Am. Phys. Soc. 34 (1993) 2107.

[8] J. Cheng, S. E. Parker, Y. Chen, D. Uzdensky, J. Comput. Phys. 245 (2013) 364.

[9] Y. Lin, X. Wang, Z. Lin, L. Chen, Plasma Phys. Controlled Fusion 47 (2005) 657.

[10] D. C. Barnes, J. Cheng, S. E. Parker, Phys. Plasmas 15 (2008) 055702.

[11] Y. Chen, S. E. Parker, Phys. Plasmas 16 (2009) 052305.

[12] D. D. Schnack, J. Cheng, D. C. Barnes, S. E. Parker, Phys. Plasmas 20 (2013) 062106.

425 [13] C. K. Birdsall, A. B. Langdon, Plasma Physics via Computer Simulation, McGraw-Hill, 1985.

[14] J. M. Dawson, Rev. Mod. Phys. 55 No. 2 (1983) 403.

427 [15] J. A. Byers, Proc. Fourth Conference on Numerical Simulations, Naval Res. Lab., Washington, D.C., 1970.

428 [16] B. D. Fried, S. D. Conte, The Plasma Dispersion Function, Academic Press, New York, 1961.

429 [17] K. E. Atkinson, An Introduction to Numerical Analysis, 2nd Edition, Wiley, 2004.

430 [18] G. Chen, L. Chacón, D. C. Barnes, J. Comput. Phys. 230 (2011) 7018.

431 [19] S. Markidis, G. Lapenta, J. Comput. Phys. 230 (2011) 7037.

432 [20] J. M. Hammersley, D. C. Handscomb, Monte Carlo Methods, Methuen, 1964

433 [21] R. Courant, K. Friedrichs, H. Lewy, Mathematische Annalen 100 (1928) 32. 